OPEN ACCESS

Edited by:

Tino Zaehle,

Otto von Guericke University

Magdeburg, Germany

Reviewed by:

Julian Koenig,

Heidelberg University, Germany

Roberta Sclocco,

Athinoula A. Martinos Center for

Biomedical Imaging and Harvard

Medical School,

United States

*Correspondence:

Dorothea D. Jenkins

jenkd@musc.edu

${ }^{\dagger}$ These authors share first authorship

Specialty section:

This article was submitted to Brain Imaging and Stimulation, a section of

the journal Frontiers in Human

Neuroscience

Received: 15 November 2019 Accepted: 20 February 2020

Published: 18 March 2020

Citation:

Badran BW, Jenkins $D D$, Cook $D$, Thompson S, Dancy M, DeVries WH, Mappin G, Summers P, Bikson $M$ and

George MS (2020) Transcutaneous Auricular Vagus Nerve Stimulation-Paired Rehabilitation for

Oromotor Feeding Problems in Newborns: An Open-Label Pilot Study.

Front. Hum. Neurosci. 14:77. doi: 10.3389/fnhum.2020.00077

\section{Transcutaneous Auricular Vagus Nerve Stimulation-Paired Rehabilitation for Oromotor Feeding Problems in Newborns: An Open-Label Pilot Study}

\author{
Bashar W. Badran ${ }^{1 \dagger}$, Dorothea D. Jenkins ${ }^{2 *}$, Daniel Cook ${ }^{1}$, Sean Thompson ${ }^{1}$, \\ Morgan Dancy ${ }^{1}$, William H. DeVries ${ }^{1}$, Georgia Mappin ${ }^{1}$, Philipp Summers ${ }^{1}$, \\ Marom Bikson ${ }^{3}$ and Mark S. George ${ }^{1,4}$
}

\begin{abstract}
${ }^{1}$ Department of Psychiatry, Medical University of South Carolina, Charleston, SC, United States, ${ }^{2}$ Department of Pediatrics, Medical University of South Carolina, Charleston, SC, United States, ${ }^{3}$ Department of Biomedical Engineering, City College of New York, New York, NY, United States, ${ }^{4}$ Ralph H. Johnson VA Medical Center, Charleston, SC, United States
\end{abstract}

Neonates born premature or who suffer brain injury at birth often have oral feeding dysfunction and do not meet oral intake requirements needed for discharge. Low oral intake volumes result in extended stays in the hospital (>2 months) and can lead to surgical implant and explant of a gastrostomy tube (G-tube). Prior work suggests pairing vagus nerve stimulation (VNS) with motor activity accelerates functional improvements after stroke, and transcutaneous auricular VNS (taVNS) has emerged as promising noninvasive form of VNS. Pairing taVNS with bottle-feeding rehabilitation may improve oromotor coordination and lead to improved oral intake volumes, ultimately avoiding the need for G-tube placement. We investigated whether taVNS paired with oromotor rehabilitation is tolerable and safe and facilitates motor learning in infants who have failed oral feeding. We enrolled 14 infants [11 premature and 3 hypoxic-ischemic encephalopathy $(\mathrm{HIE})]$ who were slated for G-tube placement in a prospective, open-label study of taVNS-paired rehabilitation to increase feeding volumes. Once-daily taVNS was delivered to the left tragus during bottle feeding for 2 weeks, with optional extension. The primary outcome was attainment of oral feeding volumes and weight gain adequate for discharge without G-tube while also monitoring discomfort and heart rate $(\mathrm{HR})$ as safety outcomes. We observed no adverse events related to stimulation, and stimulation-induced HR reductions were transient and safe and likely confirmed vagal engagement. Eight of 14 participants (57\%) achieved adequate feeding volumes for discharge without G-tube (mean treatment length: $16 \pm 6$ days). We observed significant increases in feeding volume trajectories in responders compared with pre-stimulation ( $p<0.05)$. taVNS-paired feeding rehabilitation appears safe and may improve oral feeding in infants with oromotor dyscoordination, increasing the rate of discharge without G-tube, warranting larger controlled trials.

Keywords: transcutaneous auricular vagus nerve stimulation, transcutaneous vagus nerve stimulation, vagus nerve stimulation, vagus nerve stimulation, feeding, pediatric rehabilitation, hypoxic-ischemic encephalopathy 


\section{INTRODUCTION}

In the motor task of feeding, neonates are required to coordinate a complex and rapid sequence of sucking, swallowing, and breathing, all integrated with a typical respiratory rate of 40 breaths per minute. This requires advanced sensorimotor integration of muscles of the face, head, and neck with the myelinated vagal regulation of breathing and heart rate (HR; Porges, 1992; Portales et al., 1997; Suess et al., 2000; Porges and Furman, 2011). Feeding difficulty is the primary reason for delayed hospital discharge in preterm infants with brain dysmaturation or near-term/term infants with hypoxic-ischemic encephalopathy (HIE) who are otherwise clinically stable and ready for discharge (Adamkin, 2006; Lau et al., 2015; Jackson et al., 2016). This increases hospital costs and is associated with a negative impact on long-term neurodevelopment, particularly with receptive and expressive language deficits (Adams-Chapman et al., 2013; Malas et al., 2015). The current standard of treatment for infant oromotor dysfunction consists of occupational or speech therapists feeding infants by mouth (PO) once a day to encourage safe feeding while learning this motor skill. However, many infants do not show improvement by term equivalent age, even after many weeks of rehabilitation with therapists, and have a gastrostomy tube (G-tube) placed for adequate nutrition.

Difficulty learning the motor sequence for oral feeding may be due to brain injury from infection, ischemia, and dysmaturity (Huang et al., 2015; Ismail et al., 2017). This diffuse injury results in less myelination and fewer brainstem-cortical connections (Duerden et al., 2015; Rocha-Ferreira and Hristova, 2016) and may lead to reduced corticobulbar regulation of both vagal activity and the striated muscles of the face, head, and neck (Suess et al., 2000). Atypical neural maturation with prematurity or brain injury also leads to overactive sympathetic inputs into the autonomic nervous system combined with lower parasympathetic vagal tone and persistent brainstem dysmaturity (Heilman et al., 2012; Rocha-Ferreira and Hristova, 2016). Such reactivity and neural dysmaturation make coordinating and learning a complex motor task even more difficult, explaining why the feeding mechanism must be taught through feeding rehabilitation, when it should be a normal reflex.

With improved survival rates of more critically ill neonates, the national rate of $G$-tube placement has doubled from 2000 to 2012 (Hatch et al., 2018). Complications of G-tube placement and removal often lead to subsequent hospitalizations or procedures after discharge from the nursery (McSweeney et al., 2015; Khalil et al., 2017; Hatch et al., 2018). At the Medical University of South Carolina (MUSC), preterm infants who have not reached full PO feeds by 40 -week gestational age (GA) and/or after 40 days of attempting PO feeds have a $>90 \%$ chance of eventually needing G-tube implantation to achieve full enteral feeds (Ryan and Gehle, 2019). Any therapy that facilitates motor learning and enhances feeding skills would have a significant impact for infants who fail feeding rehabilitation.

Vagus nerve stimulation (VNS) paired with motor activity enhances neuroplasticity, facilitates cortical reorganization and neurogenesis, and improves motor function post stroke (Porter et al., 2012; Engineer et al., 2015; Dawson et al., 2016). Recently, a noninvasive form of VNS known as transcutaneous auricular VNS (taVNS) targeting the auricular branch of the vagus nerve $(\mathrm{ABVN})$ has demonstrated activation of the vagal afferent and efferent networks (Kraus et al., 2013; Garcia et al., 2017; Yakunina et al., 2017; Badran et al., 2018a,c). In patients with limb impairment post stroke or brain injury, pairing taVNS with motor activation can enhance plasticity and improve functional motor recovery (Dawson et al., 2016; Pruitt et al., 2016; Redgrave et al., 2018). This human work extends the large animal literature that demonstrates pairing VNS with a behavioral intervention restores brain function (Hays et al., 2014a,b; Khodaparast et al., 2014, 2016). Therefore, both animal and adult human data support the likely efficacy of VNS-paired with motor rehabilitation.

We applied this model of taVNS paired with a motor behavior to neonates who have failed to learn the oromotor skill of feeding. We conducted a prospective, open-label trial exploring the use of once-daily taVNS-paired rehabilitation training to enhance oral feeding behavior in neonates with oromotor dyscoordination. We hypothesized that taVNS paired with bottle feeding may function in a similar mechanism by enhancing cortical plasticity in neonates with oromotor deficits, resulting in improved acquisition of the sensorimotor skill of feeding. With a favorable safety profile in adults and the ability to treat noninvasively at the bedside, taVNS is an attractive therapeutic option for neuromodulation therapies in this vulnerable population.

\section{MATERIALS AND METHODS}

\section{Study Overview}

This study was conducted at the MUSC and was approved by the MUSC Institutional Review Board. After obtaining parental consent, we enrolled 14 participants who were consulted for G-tube placement in a prospective, open-label phase 0 trial to determine the feasibility, safety, and potential clinical benefit of a novel taVNS-paired oromotor rehabilitation paradigm in neonates with oromotor dyscoordination. We reported on five of the participants in this trial in an earlier brief communication (Badran et al., 2018b). Our primary clinical outcomes were improved PO feeding volumes and attaining full PO feeds adequate for discharge, thereby avoiding G-tube implantation (Figure 1).

\section{Participants}

We included infants who were born premature at $\leq 33$ weeks' gestation at birth $(n=11)$ or suffered global HIE $(n=3)$ and who failed to make progress in PO volumes. Importantly, all enrolled participants were clinically determined to require a G-tube due to failure to achieve oral feeds sufficient for discharge from the hospital. Parents of all 14 infants had been approached about G-tube placement by the clinical teams prior to enrollment. Historically at MUSC, these infants would have $<10 \%$ chance of avoiding a G-tube. We excluded infants who were clinically unstable, were unable to attempt every feed PO, were on 


\section{Experimental Overview}

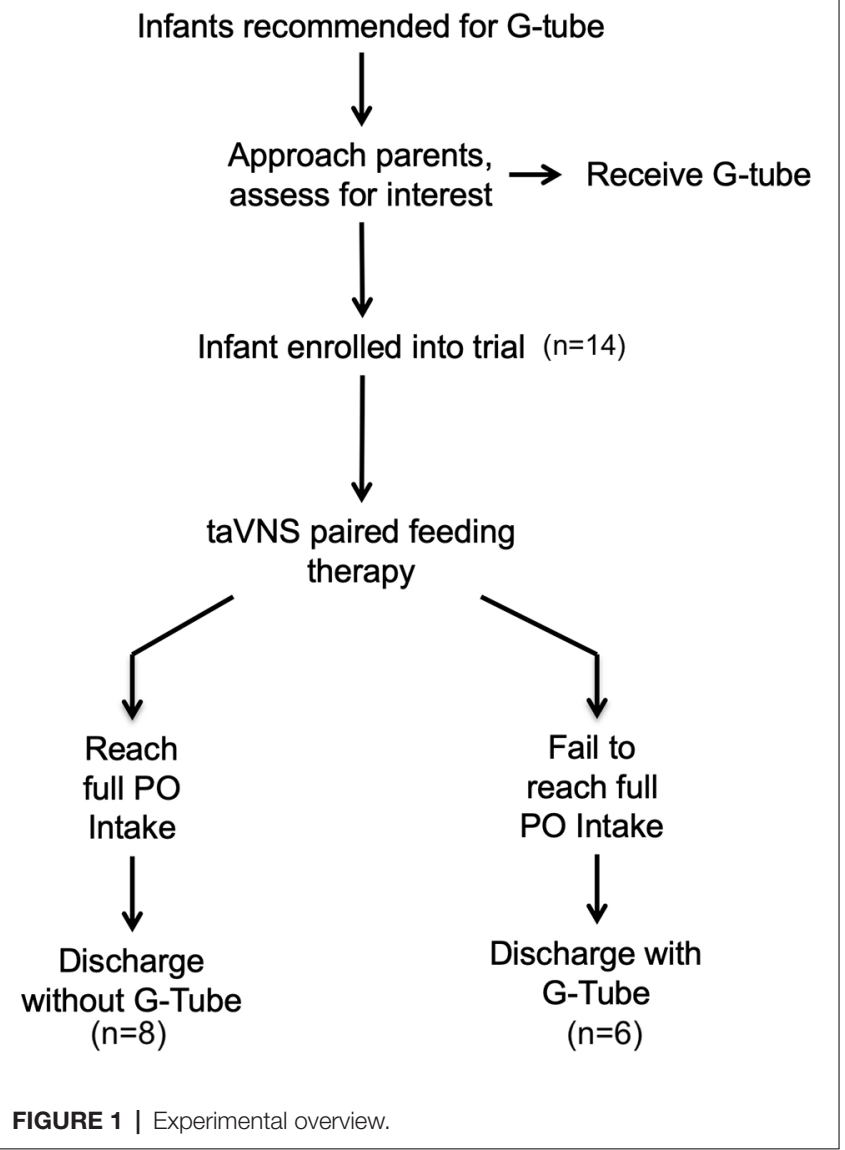

significant respiratory support with frequent bradycardia or apnea events, or had cardiomyopathy.

\section{Transcutaneous Auricular Vagus Nerve Stimulation-Paired Feeding Protocol}

We delivered taVNS once a day during a bottle feed, timed with observed sucking and swallowing for $30 \mathrm{~min}$ or the duration of the feed. Stimulation was paired with nutritive sucking and swallowing and was paused during rest or burping. The treatment period was 2 weeks, with the possibility to continue for an additional 2 weeks if substantial progress was made. If PO feeds had not progressed after 2 weeks of taVNS treatment, the parents and the clinical team made decisions about timing of G-tube placement.

\section{Transcutaneous Auricular Vagus Nerve Stimulation Setup and Technique Refinement}

We delivered taVNS using a constant current electrical nerve stimulator (Digitimer DS7AH, Digitimer LTD) connected to custom-designed neonatal ear electrodes (Figure 2). Electrodes targeted the anterior wall of the ear canal (anode) and the tragus (cathode). Stimulation was triggered manually for participants 1-7 or via a novel closed-loop electromyography (EMG) triggering system for participants 8-14 (Cook et al., 2020 under review, Brain Stimulation). The closed-loop trigger system was developed to more accurately pair stimulation trains with coordinated suck-swallow oromotor activation, to increase ease of use and to decrease operator tasks. Real-time EMG recordings were used to trigger taVNS stimulation based on masseter activation during suck-swallow. EMG leads were placed on the masseter muscle (recording), frontal eminence (reference), and center of the forehead (common).

We also refined the EMG-triggered pulse train for optimal pairing of stimulation with the sensorimotor sequence required for efficient feeding. This includes the pre-motor stage of sensing the nipple in the mouth, expressing and sensing milk on the tongue, and subsequent activation of multiple pharyngeal and hyoid muscles that effect swallowing. Many of these muscles are innervated by branches of the vagus nerve. With a 3-s pulse train following the EMG trigger, sucks that occurred at the end of the taVNS train did not receive stimulation ( $n=4$ participants). By lengthening the pulse train to $10 \mathrm{~s}$, we achieved better pairing of stimulation with suck bursts ( $n=3$ participants).

\section{Transcutaneous Auricular Vagus Nerve Stimulation Dosing} Stimulation parameters were as follows: frequency $-25 \mathrm{~Hz}$, pulse width $-500 \mu \mathrm{s}$, and current intensity $-0.1 \mathrm{~mA}$ below perceptual threshold (PT). We determined PT by increasing the stimulation current in $0.1-\mathrm{mA}$ increments while monitoring for indication that the infant perceived the stimulation, indicated by shrugging, change in facial expression, or fidgety movements. A neonatologist and a technician performed the stimulation. During treatment, infants were fed by occupational or speech therapists, staff, or parents. A custom MATLAB program recorded pulses and current intensity delivered during each session. We recorded $\mathrm{PO}$ volume intake during taVNS feed, total daily PO volume, and any adverse events.

\section{Safety Monitoring and Target Engagement}

The neonatal and infant pain scale (NIPS) scores (Lawrence et al., 1993; Witt et al., 2016) were recorded at initiation, midway, end, and $5 \mathrm{~min}$ after each treatment session. If NIPS scores increased greater than three points or the infant appeared to be sensing the stimulation, we decreased the current intensity by $0.1 \mathrm{~mA}$. We monitored redness and skin irritation at electrode site and HR on bedside monitors for bradycardia, defined per nursery protocol as $<80 \mathrm{bpm}$ for $5 \mathrm{~s}$. For target attainment, we recorded the lowest $\mathrm{HR}$ within the first $60 \mathrm{~s}$ of stimulation, the time to the lowest HR, and the rebound $\mathrm{HR}$, to verify target engagement of vagus nerve using the parasympathetic response as an indicator (Badran et al., 2018c). We also recorded HR in 60-s epochs during taVNSpaired feeds and non-stimulation (control) feeds.

\section{Primary Outcome Measures}

The primary safety outcomes were bradycardia events and NIPS score increase of greater than or equal to 3 points due to taVNS stimulation. The primary clinical outcome of this study was a binary endpoint of full oral feeds or G-tube implantation. Responders were participants who were able to increase and maintain full daily PO intake for 4 days (>120 ml/kg/day) and weight gain adequate for discharge ( $>20 \mathrm{~g} /$ day). Infants who 


\section{ABVN Innervation of the Human Auricle (He et al) ${ }^{40}$}

\section{Electrode Placement for taVNS-} Paired Feeding
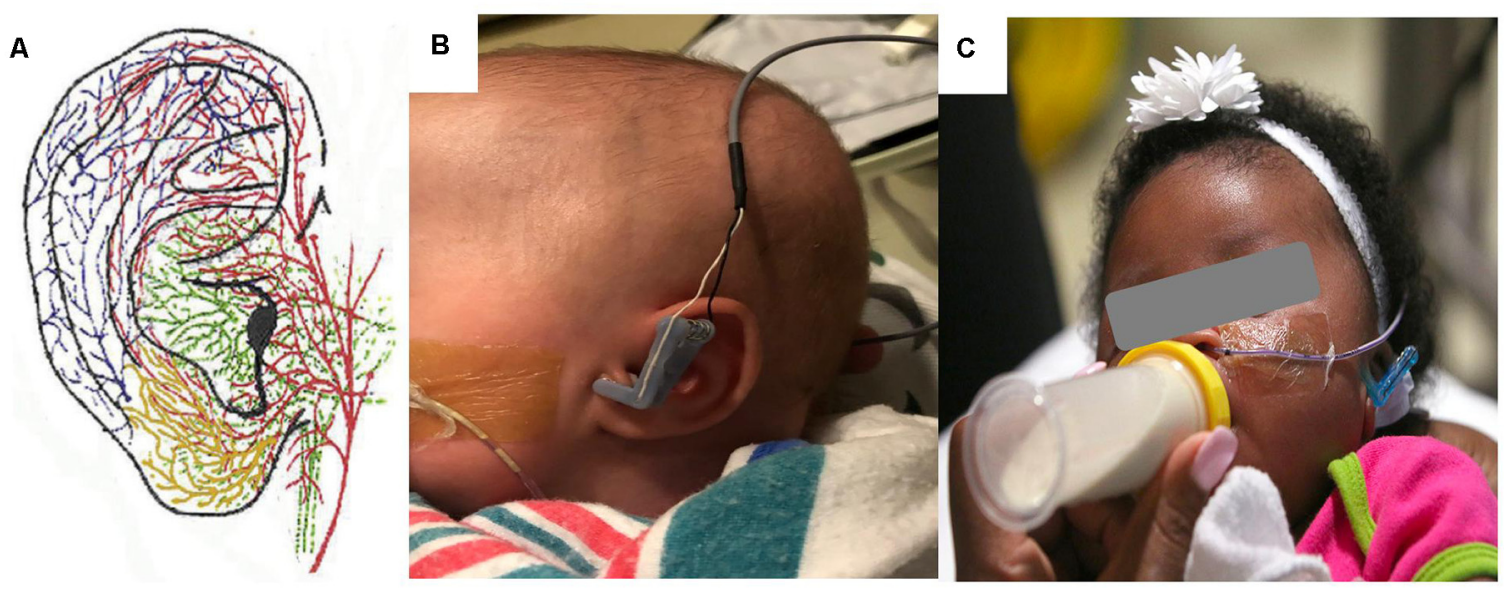

FIGURE 2 | (A) Auricular vagus nerve fibers (He et al., 2012). (B) Close-up photo of the left ear with attached custom, 3D-printed transcutaneous auricular vagus nerve stimulation (taVNS) electrodes attached. (C) Photo of the taVNS-paired feeding session with stimulation delivered concurrently with bottle feeding (written informed consent was obtained from the legal guardians for the publication of this image).

received G-tubes for inadequate intake after taVNS treatment were classified as non-responders. Other outcomes were rate of increase of daily oral feeding volumes and length of time to achieve full oral feeds.

\section{Statistical Analyses}

We analyzed group HR effects that compared HR measured before taVNS (or control) feed, the lowest HR at onset of taVNS (or control) feed, and during taVNS-paired feeding (or control) feeds using a one-way ANOVA. We then investigated the within-individual changes in HR using a paired $t$-test to compare each participant's baseline and the lowest HR prior to feed to the lowest HR during PT within taVNS or control feeds, and unpaired $t$-tests for HR differences across taVNS or control feeds. Behavioral feeding data were analyzed by comparing the slopes of the linear regression generated from the rate of daily PO volume in two different time periods: (1) the 30 days before taVNS; and (2) taVNS-paired feeding period. We compared both between- and within-group subjects in a $2 \times 2$ design (pre/post taVNS and responder/non-responder). Videofluoroscopic swallow study (VFSS) scores prior to taVNS treatment initiation were compared with treatment period according to response group via unpaired $t$-test.

\section{RESULTS}

\section{Demographics}

We enrolled 11 preterm and 3 near-term/term HIE infants. Clinical characteristics are noted in Table 1. Central nervous system (CNS) insults were prevalent (11/14) and consisted of intraventricular hemorrhage (IVH) or cerebellar hemorrhage, white matter infarction or periventricular leukomalacia (PVL), lenticulostriate vasculopathy (LSV), and acute moderate- to-severe HIE. A majority of infants (9/14) had sepsis complicating their neonatal course, which is associated with white matter neuroinflammation and infarction, and worse neurodevelopmental outcomes (Alshaikh et al., 2013; Bakhuizen et al., 2014; Bright et al., 2017; Dubner et al., 2019).

PO feeds were attempted for a mean (SD) of $49 \pm 24.3$ days before study enrollment in these 14 preterm and HIE infants. At study entry, most preterm infants were more than 44-week GA, well past term equivalent age, and had been trying to learn to feed for more than 40 days, at which point $>90 \%$ of preterm infants at MUSC have attained full PO feeds (Ryan and Gehle, 2019). Prior to enrollment in this research trial, the clinical team had approached all parents about the need for a G-tube (Figure 1).

Thirteen out of 14 infants had clinical studies of videofluoroscopic barium swallow (VFSS, $n=11$ ) or an impedance probe $(n=2)$ prior to enrollment. Six infants also had upper gastrointestinal (UGI) contrast studies. Eight infants had gastroesophageal reflux documented on one or more of these studies and were treated with histamine or proton pump antagonists. The VFSSs were performed and scored by three pediatric speech language pathologists using the Rosenbek scale (Rosenbek et al., 1996). Mean (SD) penetration and aspiration scores were $6 \pm 3$ with thin liquids (range 1-8). Six infants had maximum scores of 8 , indicating aspiration below vocal folds with no attempt to eject liquid: three of these infants were trialed with thickened feeds prior to beginning the study; two infants continued to attempt with thin maternal breast milk, which could not be adequately thickened during the taVNS treatments; one infant showed dramatic improvement in oral feeding volumes to $100 \mathrm{ml} / \mathrm{kg} /$ day after 2 weeks of taVNS treatments but had persistent coughing during feeds and was transitioned to thickened feeds near the end of the treatment course. 
TABLE 1 | Infant demographics.

\begin{tabular}{lcc}
\hline taVNS-treated infants & Preterm $(\boldsymbol{n}=\mathbf{1 1})$ & Term HIE $(\boldsymbol{n}=\mathbf{3})$ \\
\hline Sex M/F & $5 / 6$ & $0 / 3$ \\
Mean GA at birth (weeks) & $28 \pm 3$ & $36 \pm 0.5$ \\
Mean birth weight (g) & $1,027 \pm 453$ & $2,600 \pm 697$ \\
Mean GA at enrollment (weeks) & $45 \pm 5$ & $40 \pm 2$ \\
Mean days attempting PO & $57 \pm 22$ & $24 \pm 10$ \\
before taVNS & & \\
Sepsis (including NEC, & 9 & 0 \\
pneumonia, UTI, viral infections) & & 3 \\
CNS abnormalities & 8 & 1 (grade 3) \\
IVH or other intracranial bleed & 6 (grades 1 and 2) & \\
(grade) & & 2 \\
HIE (term HIE stage 2 $n=1$ ), 3 & 2 & 1 \\
(n=1); preterm HIE stages not & & 1 \\
validated) & & 1 \\
White matter infarction or PVL & 2 & 1 \\
Lenticulostriate vasculopathy & 1 & 0 \\
Infants of diabetic mothers & 3 & 0 \\
Hypoglycemia & 4 & 1 \\
Hyperglycemia & 4 & \\
Gastroesophageal reflux & 8 & \\
requiring treatment & & \\
Aspiration on MBSS & 5 &
\end{tabular}

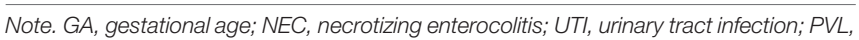
periventricular leukomalacia; taVNS, transcutaneous auricular vagus nerve stimulation; HIE, hypoxic-ischemic encephalopathy; GA, gestational age; MBSS, modified barium swallow study.

\section{Safety}

We monitored for bradycardia during both PT and during the stimulation-paired feed. There was only one bradycardia adverse event during a taVNS-paired feeding, likely unrelated to the stimulation as it was associated with choking and emesis, and readily rebounded with pausing the bottle feed.

There were no episodes of tragus irritation or redness at the electrode site. Discomfort with stimulation remained low, with the median NIPS scores [interquartile range (IQR)] of $0(0,1.0)$ before, during, and after the taVNS-paired feeding. Out of a total of 228 taVNS-paired feeding sessions, there were 10 sessions (4.3\%) during which NIPS scores increased greater than or equal to 3 points from pre-stimulation to during taVNS-paired feeding. In seven instances, the fussiness resolved quickly, and in three instances (1.3\%), stimulation current intensity was decreased for a persistent NIPS score increase. In 16 instances (7\%), we decreased stimulation when we believed it was possible that the infant was feeling stimulation but did not demonstrate a change in NIPS score. Four feeds were stopped in one infant for excessive fussiness that did not resolve after stopping stimulation, related to reflux $(\mathrm{pH}$ probe was in place for one instance).

\section{Heart Rate as a Putative Biomarker of Vagus Target Engagement}

We performed a detailed analysis of HR changes in seven consecutive participants (\#8-14), averaging 60-s HR data over the $5 \mathrm{~min}$ prior to PT and for the first $5 \mathrm{~min}$ of treatment. The mean HR before taVNS, compared with the onset of taVNS, revealed non-significant differences in physiology resulting from stimulation. The mean $\mathrm{HR}$ during the first $5 \mathrm{~min}$ before

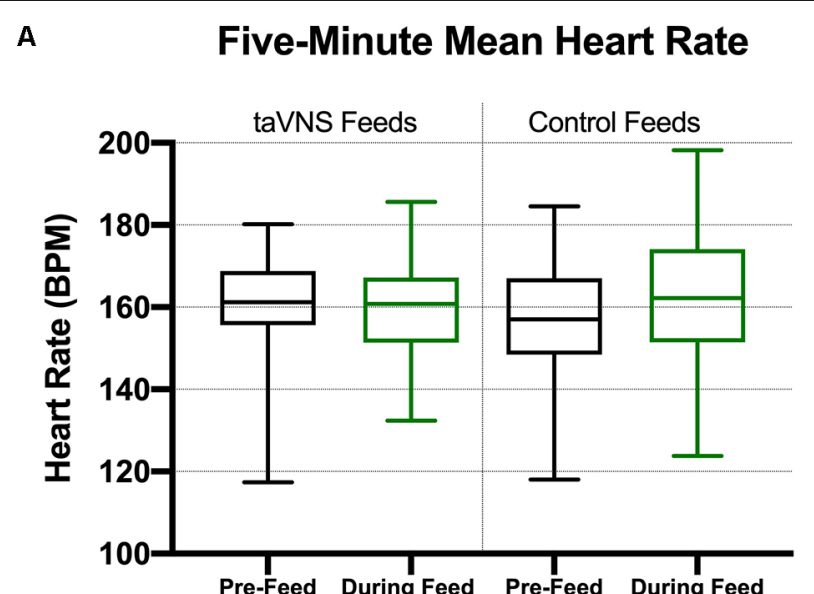

B
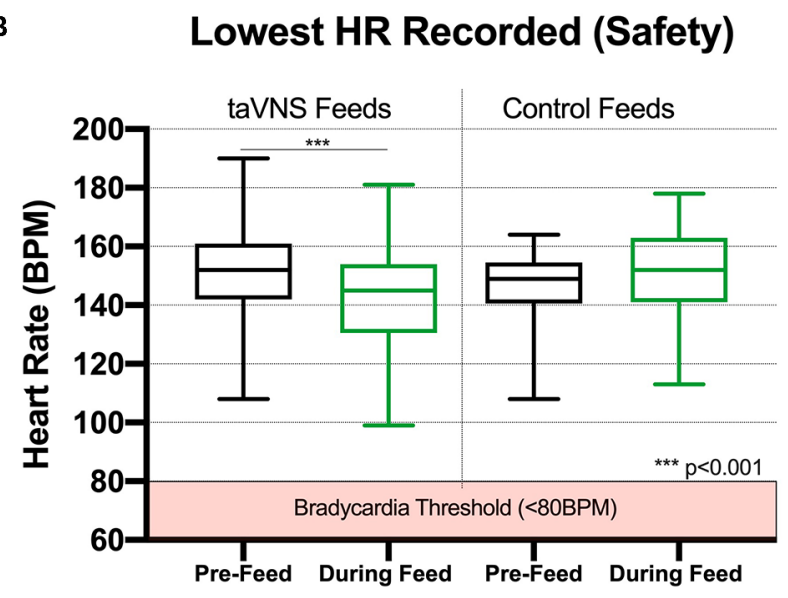

FIGURE 3 | Box and whisker plots for heart rate (HR) data collected during taVNS and control feeds (min to max). (A) taVNS with these parameters induces immediate, safe reductions in HR that recover during feeding ( $n=7$, 39 total feedings in seven participants). For the 5-min epochs prior to perceptual threshold (PT) and during taVNS-paired feeding, HR was averaged in 60-s intervals for a total of 5 min. (B) The mean lowest HR during PT was calculated from real-time $\mathrm{HR}$ monitor recorded during onset of stimulation to determine the PT. taVNS induced significant reductions in HR compared with those in pre-stimulation baseline $(p<0.0001)$; however, these reductions recovered to baseline levels immediately during the taVNS-paired feeding. There was no significant reduction in HR during control feeds (feeding HR recorded without taVNS administered, $n=7,19$ feeds).

taVNS-paired feeding was $161 \pm 11.7 \mathrm{bpm}$, compared with $159.6 \pm 11.22 \mathrm{bpm}$ during feeding ( $n=39$ feedings, seven participants, Figure 3A). For control feeds without stimulation, mean HR similarly does not change as a function of feeding. The mean HR was $156.7 \pm 15.27 \mathrm{bpm}$ for $5 \mathrm{~min}$ before the feed and $162.5 \pm 15.98 \mathrm{bpm}$ for the first 5 min during the feed, both non-significantly different from taVNS feeds.

The lowest HR was inspected as an indicator of safety profile. We compared the lowest HR before feed and the lowest HR during feeds. For the seven patients during taVNS feeds, the lowest HR before feed was $151.3 \pm 15.1$, and the lowest HR during onset of taVNS was $142.3 \pm 16.9(p=0.0005)$. For control feeds, the lowest HR prior to feed was mean (SD) of $146.2 \pm 14.6$, 
and the lowest HR during feed was mean $(\mathrm{SD})=152.5 \pm 15.7$, and we found no significant difference between timepoints (Figure 3B).

When determining the PT, we consistently observed a decrease in HR with onset of stimulation. The transient HR drop was so common and predictable that we checked impedance and the earlobe contact, and electrode position if no HR decrease was observed. During determination of PT, HR decreased a mean of $20.5 \pm 10.6 \mathrm{bpm}$ or $12.6 \pm 6.5 \%$ of the pre-taVNS HR ( $n=105$ taVNS sessions). In contrast, during control feeds, the HR decreased from before feed to the lowest HR during feed by a mean of $3.9 \pm 6.4 \mathrm{bpm}$ ( $n=19$ feeds, $n=7$ subjects). The HR decrease with onset of taVNS stimulation was significantly greater than the HR change with control feeds $(p<0.00001)$. The comparison of the lowest HR before feed and with the lowest HR with taVNS onset vs. control feeds yielded similar results (Table 3).

We observed this HR decrease as a rapid effect after taVNS onset. To determine the time frame of the HR changes, we recorded the time to the lowest $\mathrm{HR}$ and $\mathrm{HR}$ every $12 \mathrm{~s}$ during entire taVNS-paired feeds in three participants ( $n=48$ sessions). In these three participants, the mean HR decreased $16 \pm 9 \mathrm{bpm}$ or $10 \pm 3 \%$ baseline $\mathrm{HR}$ within $26 \pm 8 \mathrm{~s}$ of stimulation onset, followed by an HR rebound to or above baseline within $60 \mathrm{~s}$ from stimulation onset, which was maintained during the taVNS-paired feeding. These measurements were reproducible within and between individuals (Figures 4A-C), replicating our group's prior HR findings in an adult human taVNS study (Badran et al., 2018c).

The HR decrease was likely due to vagus target engagement, as it was significantly different than the change in HR before and during control feedings without stimulation. During non-stimulation control feedings, the HR changed by a mean of $-2.3 \pm 14.0 \mathrm{bpm}$ from HR before feed to the lowest HR during feed $(n=23, n s)$, compared with the rapid, transient mean HR decrease upon stimulation with taVNS $(-20.5 \pm 10.6 \mathrm{bpm}$, $n=104$ feeds, $p<0.00001, t$-test).

\section{Feeding Outcomes}

Of the 14 participants enrolled, who had all failed to attain feeding after an average of 49 days trying (Table 1), eight infants attained full oral feeds with weight gain adequate for discharge from the hospital after a course of taVNS-feeding paired rehabilitation (responders), and six did not receive a G-tube (non-responders). This 57\% response rate is higher than our institutional historical controls and published rates for preterm infants (Howe et al., 2007a,b; Jackson et al., 2016; Ryan and Gehle, 2019).

We examined whether the responders were starting to improve oral feeds prior to enrolling in the trial. Although there is day-to-day variability in feeding volumes, the baseline rate of change of daily $\mathrm{PO}$ volume, averaged over 5 days immediately prior to taVNS treatment, was not significantly different between responders and non-responders $(p=0.15$, Figure 5). With taVNS treatment, the rate of change of daily PO volume increased significantly in responders when compared with that in pre-treatment $(p=0.035)$. In non-responders, the
TABLE 2 | Clinical condition and treatment characteristics by responders and non-responders.

\begin{tabular}{|c|c|c|c|}
\hline taVNS-treated infants & $\begin{array}{c}\text { Responders } \\
n=8\end{array}$ & $\begin{array}{c}\text { Non-responders } \\
n=6\end{array}$ & $p$ \\
\hline $\begin{array}{l}\text { Preterm (mean GA at } \\
\text { birth, birth weight) }\end{array}$ & 6 (27 weeks, 877 g) & $\begin{array}{c}5 \text { (29 weeks, } \\
1,107 \mathrm{~g})\end{array}$ & \\
\hline Term HIE & 2 & 1 & \\
\hline Male sex & 3 & 2 & \\
\hline $\begin{array}{l}\text { Mean days attempting } \\
\text { PO pre-taVNS }\end{array}$ & $48 \pm 29$ & $49 \pm 16$ & ns \\
\hline $\begin{array}{l}\text { Mean PO volume over } \\
5 \text { days pre-taVNS }\end{array}$ & $52 \pm 22$ ml/kg/day & $45 \pm 26$ ml/kg/day & ns \\
\hline $\begin{array}{l}\text { Mean \# taVNS } \\
\text { treatments }\end{array}$ & $16 \pm 6$ & $17 \pm 3$ & ns \\
\hline $\begin{array}{l}\text { Average mA current } \\
\text { intensity }\end{array}$ & $0.82 \pm 0.2$ & $0.75 \pm 0.2$ & ns \\
\hline $\begin{array}{l}\text { Total pulses all } \\
\text { treatments }\left(10^{5}\right)\end{array}$ & $2.9 \pm 1.7$ & $2.2 \pm 0.5$ & ns \\
\hline IDM & 1 & 3 & \\
\hline $\begin{array}{l}\text { GERD requiring } \\
\text { treatment }\end{array}$ & 4 & 4 & \\
\hline $\begin{array}{l}\text { VFSS: mean (SD) PAS } \\
\text { scores }\end{array}$ & $6 \pm 3$ & $4 \pm 3$ & ns \\
\hline Aspiration on VFSS & 5 & 1 & \\
\hline Esophagitis & 1 & 2 & \\
\hline $\begin{array}{l}\text { Periventricular } \\
\text { leukomalacia }\end{array}$ & 1 & 2 & \\
\hline $\begin{array}{l}\text { Lenticulostriate } \\
\text { vasculopathy }\end{array}$ & 0 & 2 & \\
\hline
\end{tabular}

Note. taVNS, transcutaneous auricular vagus nerve stimulation; GA, gestational age; HIE, hypoxic-ischemic encephalopathy; GERD, gastroesophageal reflux disease; VFSS, videofluoroscopic swallow study; IDM, Infants of diabetic mothers; PAS, PenetrationAspiration scale.

TABLE 3 | Lowest HR for 5 min prior to and during onset of taVNS vs. control feeds ( $n=7$ subjects).

\begin{tabular}{lccl}
\hline & Lowest HR before & Lowest HR during & \\
\hline taVNS-Paired feed & $151.3 \pm 15.1$ & $142.3 \pm 16.9$ & $p=0.0005$ \\
Control feed & $146.2 \pm 14.6$ & $151.3 \pm 15.1$ & $p=0.2$ \\
\hline
\end{tabular}

Note. HR, heart rate; taVNS, transcutaneous auricular vagus nerve stimulation.

mean rate of change of daily PO feeding volumes did not change from pre-treatment to during treatment $(p=0.29)$. Responders and non-responders did not differ in the number of taVNS treatments, average current intensity, or total pulses over all treatments (Table 2).

The VFSS scores prior to taVNS treatment were not different between response groups $(p=0.3)$. Among the six infants who demonstrated aspiration below the vocal cords without effort to eject the liquid, five were responders. Of the responders with aspiration, three were taking thickened feeds prior to starting the study, two continued on thin maternal breast milk feeds with pacing, and one infant on breast milk feeds was transitioned to thickened feeds during the taVNS treatment period, after making significant progress to $100 \mathrm{ml} / \mathrm{kg} /$ day but demonstrating persistent coughing.

\section{DISCUSSION}

In this phase 0 pilot trial, one taVNS-paired feeding per day was safe and well tolerated in infants who had failed to achieve 
Heart Rate Changes During taVNS-Paired Feed

A

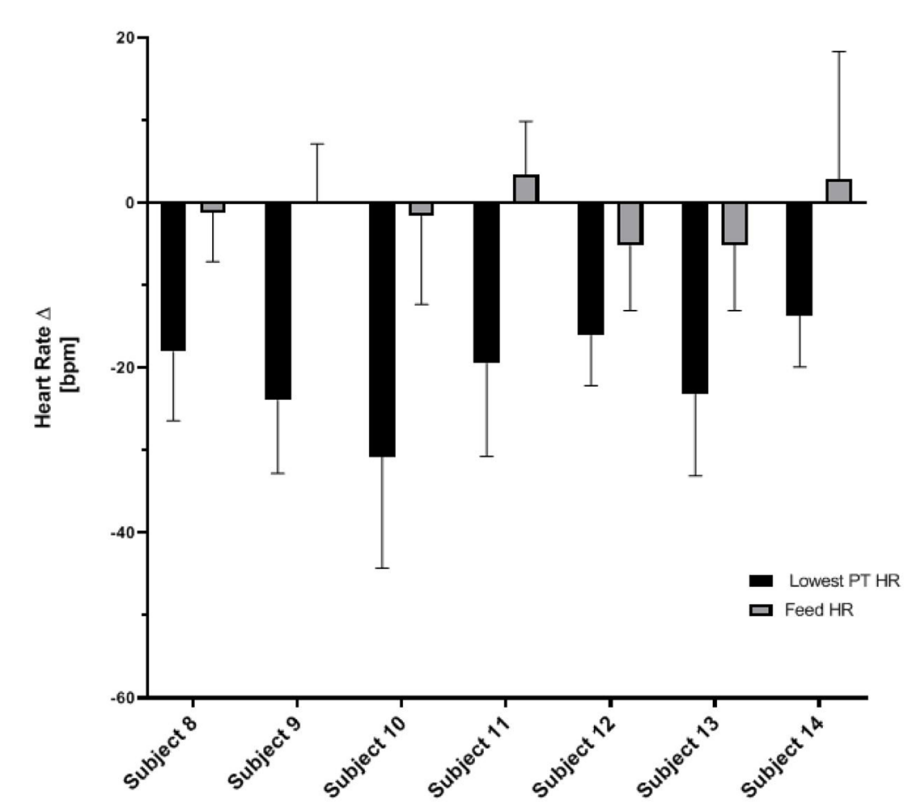

Heart Rate Changes Over

Treatment Course For Individual

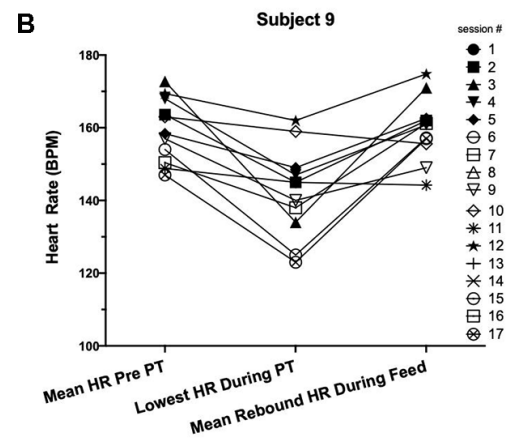

C

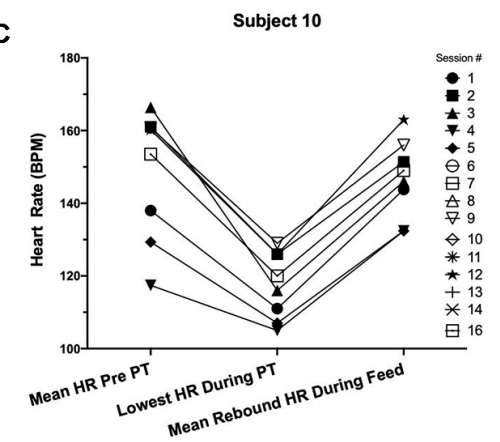

FIGURE 4 | Reproducibility and reliability of individual HR change. (A) Individual HR change from baseline with onset of stimulation and during taVNS -paired feeding by individual subject. HR is averaged over all taVNS-paired feedings for each individual subject. (B,C) HR data from individual treatment sessions in two representative participants. HR changes are shown for each individual taVNS-paired feeding session over 5 min before and during taVNS-paired feedings and the lowest HR recorded during onset of stimulation with PT determination.

full PO feeds and were referred for G-tube placement. Fiftyseven percent of treated infants were able to take all feeds by mouth within a mean of 16 days of treatment. taVNS-paired oral rehabilitation increased the likelihood of discharge without the need for G-tube implantation than did a historical retrospective comparison cohort who received standard feeding rehabilitation (Ryan and Gehle, 2019). A retrospective study of neonatal feeding outcomes at our institution built a predictive model that shows a minimal chance of reaching the required $\mathrm{PO}$ intake for discharge if the neonate has not reached $80 \mathrm{ml} / \mathrm{kg} /$ day by the 20th day attempting PO (Ryan and Gehle, 2019). Our cohort attempted PO for a mean of $49 \pm 24.3$ days prior to taVNS treatment, for which the in-house model predicts a spontaneous recovery rate of less than $10 \%$.

Comparable published data indicate that preterm infants born at 25-32 weeks' gestation, with respiratory complications of bronchopulmonary dysplasia, attained full oral feeds at $38.5 \pm 2.8$ weeks' gestation, whereas those without BPD did so at $35.5 \pm 1.7$ weeks' gestation (Howe et al., 2007b). In one large retrospective study of 560 preterm infants born at 32-36 week's gestation, the median time to achieve full oral feeds was 12 days overall (Jackson et al., 2016). Although our taVNS-paired feeding study was non-randomized, our rate of successful attainment of full oral feeds is promising when compared with our historical control data as well as those from other observational studies.
Infants must develop oromotor skills of "suck-swallow" coordination in a particular sequence in order to feed effectively (Lau et al., 2003). Postmature infants (>41-week GA) who were born preterm and are not able to take full feeds by mouth may not be able to start oral feeds during a critical developmental window of oromotor neuroplasticity for learning feeding motor skills (Huang et al., 2015; Ismail et al., 2017). Sick newborns of term age (37- to 41-week GA) who have had critical illnesses frequently do not exhibit a suck-swallow reflex for feeding and may also have to learn this motor sequence, similar to preterm infants.

Both preterm and HIE infants suffer brain injury, triggering excessive stimulation of inflammatory pathways, impairing normal developmental functions of directing neuronal integration and foundational brain circuitry. After birth, the cortex and basal ganglia (BG) undergo significant integrative connectivity associated with shaping of central motor pathways. Disturbance of these processes leads to abnormal connections (Rocha-Ferreira and Hristova, 2016), and along with decreased populations of myelinating cells and inter-neurons, results in brain dysmaturity in preterm infants (Duerden et al., 2015) or overt brain injury in term HIE infants and subsequent motor, cognitive, and neurobehavioral impairments (Rocha-Ferreira and Hristova, 2016). Although postnatally the developing brain is more plastic than the adult brain and thus might be expected to 


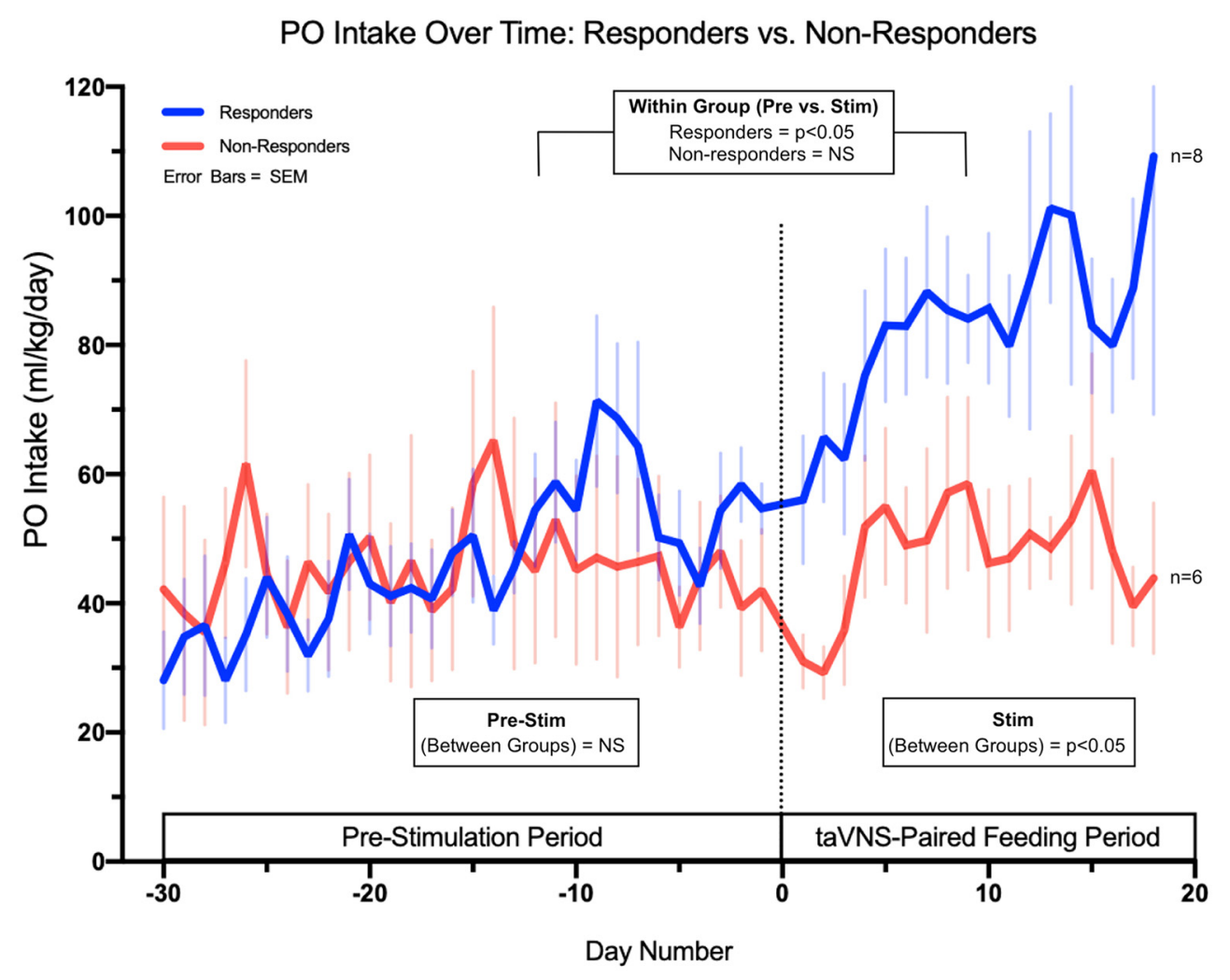

FIGURE 5 | Daily PO intake feeding volumes in ml/kg/day for both responders (full PO feeds without G-tube) and non-responders (G-tube placement). The data demonstrate no significant difference in feeding trajectories between groups in the pre-stimulation phase but significant differences in trajectories between responders and non-responders upon initiation of taVNS-paired feeding.

have better recovery following injury, we engage the developing brain to a very limited extent with clinical rehabilitation therapy while infants are still in the nursery.

The data in both animals and adult humans are convincing in that VNS paired with a stimulus improves functioning (Porter et al., 2012; Khodaparast et al., 2013, 2014, 2016; Dawson et al., 2016; Capone et al., 2017; Meyers et al., 2018). In our neonates with brain dysmaturity or overt brain injury, neuromodulation of abnormal circuits may positively influence neuronal connectivity and neuroplasticity (Kilgard, 2012; Meyers et al., 2018). If we can influence the circuitry early, before motor patterns are fixed, we may improve the developmental deficits that these children experience, starting with feeding delays in the nursery. With approximately 380,000 preterm infants and 4,000 term infants with HIE born in the USA per year, this therapy may translate to a large number of infants and have major impact on their outcomes.

Decreasing G-tube placement and length of stay due to feeding delays is a significant, longer-term goal of these studies. The time to attain full feeds accounted for $90 \%$ of variance in length of stay in two reports (Adamkin, 2006; Jackson et al., 2016). Earlier discharge without a G-tube may reduce medical complications for the patients and decrease costs, while offering substantial benefit to families waiting to bring their children home. Beyond feeding and earlier discharge, reinforced plasticity of oromotor function may impact short- and long-term neurodevelopment, particularly language skills.

Even late preterm infants can have difficulty learning to feed. In one retrospective study, one third of 35-36 weeks' gestation infants had feeding problems, and $76 \%$ of these had delayed discharge due to poor oromotor coordination (Wang et al., 2004). Also, males may have more difficulty as they have been shown to have later emergence of oral-lingual movements and pharyngeal activity than do females (Miller et al., 2006). In our study, we limited inclusion to preterm infants less than or equal to 33-week GA at birth or near-term to term infants with HIE. In the future, we need to enroll infants of wider GAs, conduct randomized controlled trials with sham stimulation, and enroll sufficient numbers for evaluation of sex differences.

Moreover, if taVNS is successful in the targeted motor behavior of feeding, we will extend investigation of early neuromodulatory therapy in high-risk infants to prevent or mitigate other life-long motor problems, such as cerebral palsy. This taVNS pilot trial, the first in human neonates, may provide a foundation for application of these therapies to infants at high risk for motor problems who have few alternative treatments.

Our HR data suggest that taVNS engages the vagal parasympathetic system. A rapid, transient HR decrease of $12 \%$ from baseline at the onset of taVNS stimulation was reproducible and reliable. The observed HR change suggests vagus nerve target 
engagement by taVNS-paired feeding in neonates and may be useful in multi-day trials.

Vagal efferent HR change is useful as a stimulation indicator if it is not harmful. Safety is the foremost consideration for treatments in vulnerable infants who are premature or have suffered HIE, who have often recovered from significant respiratory, cardiovascular, or CNS conditions. We did not observe adverse effects of bradycardia or rebound tachycardia that were solely related to stimulation, or any other adverse effects. Mean neonatal discomfort scores measured with the NIPS did not significantly increase during stimulation in over 200 treatment sessions, and we decreased current intensity in only three instances for possible stimulation-induced increase in NIPS scores. No parent withdrew their infant from the study, and parents and staff accepted the treatment as well as tolerated by the infants.

It is unclear why some participants did not respond. Systemic and neuro-inflammation or medications that inhibit synaptic plasticity and learning (e.g., mineralocorticoid receptor inhibitors) may impair learning this motor skill (Favrais et al., 2011; Kuban et al., 2014; Leviton et al., 2016; Kelley et al., 2017). Alternatively, other negative sensory inputs of reflux or esophagitis may counteract positive sensorimotor circuit stimulation (Wingenfeld and Otte, 2019). Half of the non-responders (3/6) and only one of eight responders were infants of diabetic mothers with poor glucose control during pregnancy, which induces a pro-oxidative state for the mother and fetus known to be associated with immune activation, endothelial cell injury, and worse fetal and neonatal outcomes (Teodoro et al., 2013; Durga et al., 2018). A larger sample size may identify specific predictors for responder status leading to a decrease in variance in treatment response.

Further investigation and refinement of treatment parameters will likely also improve treatment response. Although these stimulation parameters were partially optimized in adults and to some extent in this trial, the potential responsiveness of specific circuits is likely determined by the sum as well as timing of stimulatory and inhibitory impulses. For example, we initially tested a pulse-train length of $3 \mathrm{~s}$ with the EMG closed-loop system, which proved too short for rhythmic suck-swallow sequences in some infants. We then increased to a 10-s train with each EMG-driven stimulation.

\section{LIMITATIONS}

The taVNS methodology was adjusted over the course of this study to more closely pair stimulation with feeding (Cook et al., 2020 in press Brain Stimulation). In participants $1-7$, researchers activated taVNS manually when the infant was seen to be actively feeding; in participants 8-14, an EMG triggered taVNS-paired stimulation to sucking motor function. The latter would be expected to have better feeding outcomes, if learning depends on the precision of timing the stimulation with the motor activity. Over the study, we also developed several different ear electrodes to maximize contact and minimize the need for readjustment during feeds. All were confirmed by $\mathrm{HR}$ and resistance tests to deliver current to the tragus. We did not perform VFSS on every baby and did not explore the physiologic impairments in swallowing or causes of penetration/aspiration in this pilot trial. In future studies, we intend to document specific changes in swallowing function before and after taVNS-paired feeding treatment using a more precise scale adapted for infants (Martin-Harris et al., 2019). We also did not perform sham stimulation, to compare the lowest HR during sham and active taVNS stimulation. However, the HR changes with feeds in the control group indicate that HR usually increases during feeding, compared with the rapid decrease seen with PT in the taVNS feeds.

\section{CONCLUSION}

This is the first study investigating taVNS paired with the motor sequence of suck, swallow, and breath to potentially enhance oromotor learning in neonates and infants. In infants who had failed oromotor rehabilitative feeding techniques by therapists, taVNS treatments resulted in 57\% achieving full oral feeds adequate for discharge without needing a G-tube. Further, taVNS appeared safe in neonates and infants with no adverse effects. Target engagement may be determined at each session by a brief HR decrease. Further investigations and a randomized trial are needed to confirm our promising results of improved feeding outcomes in infants treated with taVNS.

\section{DATA AVAILABILITY STATEMENT}

The datasets generated for this study are available on request to the corresponding author.

\section{ETHICS STATEMENT}

The studies involving human participants were reviewed and approved by MUSC IRB 1. Written informed consent to participate in this study was provided by the participants' legal guardian/next of kin.

\section{AUTHOR CONTRIBUTIONS}

$\mathrm{BB}, \mathrm{DJ}, \mathrm{DC}, \mathrm{MD}, \mathrm{WD}, \mathrm{GM}, \mathrm{PS}, \mathrm{ST}, \mathrm{MB}$, and $\mathrm{MG}$ all made substantial contributions to the conception and design, acquisition of data, or analysis and interpretation of data and participated in drafting, editing, and final approval of this manuscript.

\section{FUNDING}

This study was funded by the National MUSC Center of Neuromodulation for Rehabilitation (NC NM4R) and COBRE for Stroke Recovery. The National Center of Neuromodulation for Rehabilitation (NC NM4R) is supported by the Eunice Kennedy Shriver National Institute of Child Health \& Human Development of the National Institutes of Health under award number P2CHD086844 and P20GM109040. 


\section{REFERENCES}

Adamkin, D. H. (2006). Feeding problems in the late preterm infant. Clin. Perinatol. 33, 831-837. doi: 10.1016/j.clp.2006.09.003

Adams-Chapman, I., Bann, C. M., Vaucher, Y. E., Stoll, B. J., and Eunice Kennedy Shriver National Institute of Child Health and Human Development Neonatal Research Network. (2013). Association between feeding difficulties and language delay in preterm infants using Bayley Scales of Infant DevelopmentThird Edition. J. Pediatr. 163, 680.e1-3-685.e1-3. doi: 10.1016/j.jpeds.2013. 03.006

Alshaikh, B., Yusuf, K., and Sauve, R. (2013). Neurodevelopmental outcomes of very low birth weight infants with neonatal sepsis: systematic review and metaanalysis. J. Perinatol. 33, 558-564. doi: 10.1038/jp.2012.167

Badran, B. W., Dowdle, L. T., Mithoefer, O. J., LaBate, N. T., Coatsworth, J., Brown, J. C., et al. (2018a). Neurophysiologic effects of transcutaneous auricular vagus nerve stimulation (taVNS) via electrical stimulation of the tragus: a concurrent taVNS/fMRI study and review. Brain Stimul. 11, 492-500. doi: 10.1016/j.brs.2017.12.009

Badran, B. W., Jenkins, D. D., DeVries, W. H., Dancy, M., Summers, P. M., Mappin, G. M., et al. (2018b). Transcutaneous auricular vagus nerve stimulation (taVNS) for improving oromotor function in newborns. Brain Stimul. 11, 1198-1200. doi: 10.1016/j.brs.2018.06.009

Badran, B. W., Mithoefer, O. J., Summer, C. E., LaBate, N. T., Glusman, C. E., Badran, A. W., et al. (2018c). Short trains of transcutaneous auricular vagus nerve stimulation (taVNS) have parameter-specific effects on heart rate. Brain Stimul. 11, 699-708. doi: 10.1016/j.brs.2018.04.004

Bakhuizen, S. E., de Haan, T. R., Teune, M. J., van Wassenaer-Leemhuis, A. G., van der Heyden, J. L., van der Ham, D. P., et al. (2014). Meta-analysis shows that infants who have suffered neonatal sepsis face an increased risk of mortality and severe complications. Acta Paediatr. 103, 1211-1218. doi: 10.1111/apa.12764

Bright, H. R., Babata, K., Allred, E. N., Erdei, C., Kuban, K. C. K., Joseph, R. M., et al. (2017). Neurocognitive outcomes at 10 years of age in extremely preterm newborns with late-onset bacteremia. J. Pediatr. 187, 43.e1-49.e1. doi: 10.1016/j.jpeds.2017.04.045

Capone, F., Miccinilli, S., Pellegrino, G., Zollo, L., Simonetti, D., Bressi, F., et al. (2017). Transcutaneous vagus nerve stimulation combined with robotic rehabilitation improves upper limb function after stroke. Neural Plast. 2017:7876507. doi: 10.1155/2017/7876507

Cook, D. N., Thompson, S., Stomberg-Firestein, S., Bikson, M., George, M. S., Jenkins, D. D., et al (2020). Design and validation of a closed-loop, motor-activated auricular vagus nerve stimulation (MAAVNS) system for neurorehabilitation. Brain Stimulation. doi: 10.1016/j.brs.2020.02.028

Dawson, J., Pierce, D., Dixit, A., Kimberley, T. J., Robertson, M., Tarver, B., et al. (2016). Safety, feasibility, and efficacy of vagus nerve stimulation paired with upper-limb rehabilitation after ischemic stroke. Stroke 47, 143-150. doi: 10.1161 /strokeaha.115.010477

Dubner, S. E., Dodson, C. K., Marchman, V. A., Ben-Shachar, M., Feldman, H. M., and Travis, K. E. (2019). White matter microstructure and cognitive outcomes in relation to neonatal inflammation in 6-year-old children born preterm. Neuroimage Clin. 23:101832. doi: 10.1016/j.nicl.2019.101832

Duerden, E. G., Foong, J., Chau, V., Branson, H., Poskitt, K. J., Grunau, R. E., et al. (2015). Tract-based spatial statistics in preterm-born neonates predicts cognitive and motor outcomes at 18 months. AJNR Am. J. Neuroradiol. 36, 1565-1571. doi: 10.3174/ajnr.a4312

Durga, K. D., Adhisivam, B., Vidya, G., Vishnu Bhat, B., Bobby, Z., and Chand, P. (2018). Oxidative stress and DNA damage in newborns born to mothers with hyperglycemia - a prospective cohort study. J. Matern. Fetal Neonatal Med. 31, 2396-2401. doi: 10.1080/14767058.2017.1344630

Engineer, C. T., Engineer, N. D., Riley, J. R., Seale, J. D., and Kilgard, M. P. (2015). Pairing speech sounds with vagus nerve stimulation drives stimulus-specific cortical plasticity. Brain Stimul. 8, 637-644. doi: 10.1016/j.brs.2015.01.408

Favrais, G., van de Looij, Y., Fleiss, B., Ramanantsoa, N., Bonnin, P., StoltenburgDidinger, G., et al. (2011). Systemic inflammation disrupts the developmental program of white matter. Ann. Neurol. 70, 550-565. doi: 10.1002/ana.22489

Garcia, R. G., Lin, R. L., Lee, J., Kim, J., Barbieri, R., Sclocco, R., et al. (2017). Modulation of brainstem activity and connectivity by respiratory-gated auricular vagal afferent nerve stimulation (RAVANS) in migraine patients. Pain 158, 1461-1472. doi: 10.1097/j.pain.0000000000000930
Hatch, L. D., Scott, T. A., Walsh, W. F., Goldin, A. B., Blakely, M. L., and Patrick, S. W. (2018). National and regional trends in gastrostomy in very low birth weight infants in the USA: 2000-2012. J. Perinatol. 38, 1270-1276. doi: 10.1038/s41372-018-0145-4

Hays, S. A., Khodaparast, N., Hulsey, D. R., Ruiz, A., Sloan, A. M., Rennaker, R. L., et al. (2014a). Vagus nerve stimulation during rehabilitative training improves functional recovery after intracerebral hemorrhage. Stroke 45, 3097-3100. doi: 10.1161/strokeaha.114.006654

Hays, S. A., Khodaparast, N., Ruiz, A., Sloan, A. M., Hulsey, D. R., Rennaker, R. L., et al. (2014b). The timing and amount of vagus nerve stimulation during rehabilitative training affect poststroke recovery of forelimb strength. Neuroreport 25, 676-682. doi: 10.1097/wnr.0000000000000154

He, W., Wang, X., Shi, H., Shang, H., Li, L., Jing, X., et al. (2012). Auricular acupuncture and vagal regulation. Evid. Based Complement. Alternat. Med. 2012:786839. doi: 10.1155/2012/786839

Heilman, K. J., Connolly, S. D., Padilla, W. O., Wrzosek, M. I., Graczyk, P. A., and Porges, S. W. (2012). Sluggish vagal brake reactivity to physical exercise challenge in children with selective mutism. Dev. Psychopathol. 24, 241-250. doi: $10.1017 /$ s0954579411000800

Howe, T. H., Sheu, C. F., Hinojosa, J., Lin, J., and Holzman, I. R. (2007a). Multiple factors related to bottle-feeding performance in preterm infants. Nurs. Res. 56, 307-311. doi: 10.1097/01.nnr.0000289498.99542.dd

Howe, T. H., Sheu, C. F., and Holzman, I. R. (2007b). Bottle-feeding behaviors in preterm infants with and without bronchopulmonary dysplasia. Am. J. Occup. Ther. 61, 378-383. doi: 10.5014/ajot.61.4.378

Huang, X., Stodieck, S. K., Goetze, B., Cui, L., Wong, M. H., Wenzel, C., et al. (2015). Progressive maturation of silent synapses governs the duration of a critical period. Proc. Natl. Acad. Sci. U S A 112, E3131-E3140. doi: 10.1073/pnas.1506488112

Ismail, F. Y., Fatemi, A., and Johnston, M. V. (2017). Cerebral plasticity: windows of opportunity in the developing brain. Eur. J. Paediatr. Neurol. 21, 23-48. doi: 10.1016/j.ejpn.2016.07.007

Jackson, B. N., Kelly, B. N., McCann, C. M., and Purdy, S. C. (2016). Predictors of the time to attain full oral feeding in late preterm infants. Acta Paediatr. 105, e1-e6. doi: 10.1111/apa.13227

Kelley, M. H., Wu, W. W., Lei, J., McLane, M., Xie, H., Hart, K. D., et al. (2017). Functional changes in hippocampal synaptic signaling in offspring survivors of a mouse model of intrauterine inflammation. J. Neuroinflammation 14:180 doi: 10.1186/s12974-017-0951-1

Khalil, S. T., Uhing, M. R., Duesing, L., Visotcky, A., Tarima, S., and NghiemRao, T. H. (2017). Outcomes of infants with home tube feeding: comparing nasogastric vs. gastrostomy tubes. JPEN J. Parenter. Enteral Nutr. 41, 1380-1385. doi: 10.1177/0148607116670621

Khodaparast, N., Hays, S. A., Sloan, A. M., Fayyaz, T., Hulsey, D. R., Rennaker, R. L., et al. (2014). Vagus nerve stimulation delivered during motor rehabilitation improves recovery in a rat model of stroke. Neurorehabil. Neural Repair 28, 698-706. doi: 10.1177/1545968314521006

Khodaparast, N., Hays, S. A., Sloan, A. M., Hulsey, D. R., Ruiz, A., Pantoja, M. et al. (2013). Vagus nerve stimulation during rehabilitative training improves forelimb strength following ischemic stroke. Neurobiol. Dis. 60, 80-88. doi: 10.1016/j.nbd.2013.08.002

Khodaparast, N., Kilgard, M. P., Casavant, R., Ruiz, A., Qureshi, I., Ganzer, P. D., et al. (2016). Vagus nerve stimulation during rehabilitative training improves forelimb recovery after chronic ischemic stroke in rats. Neurorehabil. Neural Repair 30, 676-684. doi: 10.1177/1545968315616494

Kilgard, M. P. (2012). Harnessing plasticity to understand learning and treat disease. Trends Neurosci. 35, 715-722. doi: 10.1016/j.tins.2012.09.002

Kraus, T., Kiess, O., Hösl, K., Terekhin, P., Kornhuber, J., and Forster, C. (2013). CNS BOLD fMRI effects of sham-controlled transcutaneous electrical nerve stimulation in the left outer auditory canal-a pilot study. Brain Stimul. 6, 798-804. doi: 10.1016/j.brs.2013.01.011

Kuban, K. C., O’Shea, T. M., Allred, E. N., Paneth, N., Hirtz, D., Fichorova, R. N., et al. (2014). Systemic inflammation and cerebral palsy risk in extremely preterm infants. J. Child Neurol. 29, 1692-1698. doi: 10.1177/0883073813513335

Lau, C., Bhat, K., Potak, D., and Schanler, R. J. (2015). Oral feeding assessment predicts length of hospital stay in late preterm infants. J. Pediatr. Mother Care 1:102. doi: 10.19104/japm.2016.102 
Lau, C., Smith, E. O., and Schanler, R. J. (2003). Coordination of suck-swallow and swallow respiration in preterm infants. Acta Paediatr. 92, 721-727. doi: 10.1111/j.1651-2227.2003.tb00607.x

Lawrence, J., Alcock, D., McGrath, P., Kay, J., MacMurray, S. B., and Dulberg, C. (1993). The development of a tool to assess neonatal pain. Neonatal Netw. 12, 59-66.

Leviton, A., Allred, E. N., Fichorova, R. N., Kuban, K. C., Michael O'Shea, T., Dammann, O., et al. (2016). Systemic inflammation on postnatal days 21 and 28 and indicators of brain dysfunction 2 years later among children born before the 28th week of gestation. Early Hum. Dev. 93, 25-32. doi: 10.1016/j. earlhumdev.2015.11.004

Malas, K., Trudeau, N., Chagnon, M., and McFarland, D. H. (2015). Feedingswallowing difficulties in children later diagnosed with language impairment. Dev. Med. Child Neurol. 57, 872-879. doi: 10.1111/dmcn.12749

Martin-Harris, B., Carson, K. A., Pinto, J. M., and Lefton-Greif, M. A. (2019). $\operatorname{BaByVFSSImP}((\mathrm{c}))$ a novel measurement tool for videofluoroscopic assessment of swallowing impairment in bottle-fed babies: establishing a standard. Dysphagia 35, 90-98. doi: 10.1007/s00455-01910008-x

McSweeney, M. E., Kerr, J., Jiang, H., and Lightdale, J. R. (2015). Risk factors for complications in infants and children with percutaneous endoscopic gastrostomy tubes. J. Pediatr. 166, 1514.e1-1519.e1. doi: 10.1016/j.jpeds.2015. 03.009

Meyers, E. C., Solorzano, B. R., James, J., Ganzer, P. D., Lai, E. S., Rennaker, R. L., et al. (2018). Vagus nerve stimulation enhances stable plasticity and generalization of stroke recovery. Stroke 49, 710-717. doi: 10.1161/strokeaha. 117.019202

Miller, J. L., Macedonia, C., and Sonies, B. C. (2006). Sex differences in prenatal oral-motor function and development. Dev. Med. Child Neurol. 48, 465-470. doi: 10.1017/s0012162206001009

Porges, S. W. (1992). Vagal tone: a physiologic marker of stress vulnerability. Pediatrics 90, 498-504.

Porges, S. W., and Furman, S. A. (2011). The early development of the autonomic nervous system provides a neural platform for social behavior: a polyvagal perspective. Infant Child Dev. 20, 106-118. doi: 10.1002/icd.688

Portales, A. L., Porges, S. W., Doussard-Roosevelt, J. A., Abedin, M., Lopez, R., Young, M. A., et al. (1997). Vagal regulation during bottle feeding in low-birthweight neonates: support for the gustatory-vagal hypothesis. Dev. Psychobiol. 30, 225-233. doi: 10.1002/(sici)1098-2302(199704)30:3<225::aiddev5 > 3.0.co; $2-\mathrm{r}$

Porter, B. A., Khodaparast, N., Fayyaz, T., Cheung, R. J., Ahmed, S. S., Vrana, W. A., et al. (2012). Repeatedly pairing vagus nerve stimulation with a movement reorganizes primary motor cortex. Cereb. Cortex 22, 2365-2374. doi: 10.1093/cercor/bhr316

Pruitt, D. T., Schmid, A. N., Kim, L. J., Abe, C. M., Trieu, J. L., Choua, C., et al. (2016). Vagus nerve stimulation delivered with motor training enhances recovery of function after traumatic brain injury. J. Neurotrauma 33, 871-879. doi: $10.1089 /$ neu.2015.3972
Redgrave, J. N., Moore, L., Oyekunle, T., Ebrahim, M., Falidas, K., Snowdon, N., et al. (2018). Transcutaneous auricular vagus nerve stimulation with concurrent upper limb repetitive task practice for poststroke motor recovery: a pilot study. J. Stroke Cerebrovasc. Dis. 27, 1998-2005. doi: 10.1016/j.jstrokecerebrovasdis. 2018.02.056

Rocha-Ferreira, E., and Hristova, M. (2016). Plasticity in the neonatal brain following hypoxic-ischaemic injury. Neural Plast. 2016:4901014. doi: 10.1155/2016/4901014

Rosenbek, J. C., Robbins, J. A., Roecker, E. B., Coyle, J. L., and Wood, J. L. (1996). A penetration-aspiration scale. Dysphagia 11, 93-98. doi: 10.1007/bf00417897

Ryan, R. M. R. V., and Gehle, D. (2019). "Can we predict who will need a feeding gastrostomy tube in premature babies at discharge from the NICU?" in Pedaitric Academic Societies meeting (Baltimore).

Suess, P. E., Alpan, G., Dulkerian, S. J., Doussard-Roosevelt, J., Porges, S. W., and Gewolb, I. H. (2000). Respiratory sinus arrhythmia during feeding: a measure of vagal regulation of metabolism, ingestion and digestion in preterm infants. Dev. Med. Child Neurol. 42, 169-173. doi: 10.1017/s001216220000030x

Teodoro, J. S., Gomes, A. P., Varela, A. T., Duarte, F. V., Rolo, A. P., and Palmeira, C. M. (2013). Uncovering the beginning of diabetes: the cellular redox status and oxidative stress as starting players in hyperglycemic damage. Mol. Cell. Biochem. 376, 103-110. doi: 10.1007/s11010-012-1555-9

Wang, M. L., Dorer, D. J., Fleming, M. P., and Catlin, E. A. (2004). Clinical outcomes of near-term infants. Pediatrics 114, 372-376. doi: 10.1542/peds.114. 2.372

Wingenfeld, K., and Otte, C. (2019). Mineralocorticoid receptor function and cognition in health and disease. Psychoneuroendocrinology 105, 25-35. doi: 10.1016/j.psyneuen.2018.09.010

Witt, N., Coynor, S., Edwards, C., and Bradshaw, H. (2016). A guide to pain assessment and management in the neonate. Curr. Emerg. Hosp. Med. Rep. 4, 1-10. doi: 10.1007/s40138-016-0089-y

Yakunina, N., Kim, S. S., and Nam, E. C. (2017). Optimization of transcutaneous vagus nerve stimulation using functional MRI. Neuromodulation 20, 290-300. doi: $10.1111 /$ ner. 12541

Conflict of Interest: BB, DJ, DC and MG have pending patents on the methods described in this manuscript.

The remaining authors declare that the research was conducted in the absence of any commercial or financial relationships that could be construed as a potential conflict of interest.

Copyright (C) 2020 Badran, Jenkins, Cook, Thompson, Dancy, DeVries, Mappin, Summers, Bikson and George. This is an open-access article distributed under the terms of the Creative Commons Attribution License (CC BY). The use, distribution or reproduction in other forums is permitted, provided the original author(s) and the copyright owner(s) are credited and that the original publication in this journal is cited, in accordance with accepted academic practice. No use, distribution or reproduction is permitted which does not comply with these terms. 